Bundesgesundheitsbl 2020 - 63:657-659 https://doi.org/10.1007/s00103-020-03117-8 Published online: 11 March 2020 (c) Springer-Verlag GmbH Deutschland, ein Teil von Springer Nature 2020
The Guideline of the German Association for the Control of Virus Diseases (DVV) and the Robert Koch Institute (RKI) for testing chemical disinfectants regarding their virucidal efficacy within the field of human medicine, published in the journal Bundesgesundheitsblatt in the version of December 1st, 2014 [1] described the use of the large volume plating method for determination of disinfectant efficacy for the first time. In order to take account of resulting questions, the application and conduct as well as the determination of the results shall be explained in detail hereafter.
This article is a translation of the German announcement 2. Mitteilung des DVV/GFV-Fachausschusses Virusdesinfektion zur DVV/RKI-Leitlinie in der Fassung vom 01.12.2014. Erläuterung zur Bedeutung, Anwendung und Berechnung des "Large-Volume-Platings" (LVP). Bundesgesundheitsbl 2016·59:540-542. https://doi.org/10.1007/s00103-016-2325-8

Holger F. Rabenau' · Ingeborg Schwebke ${ }^{2}$. Johannes Blümel ${ }^{3} \cdot$ Maren Eggers $^{4}$. Ingrid Rapp ${ }^{5}$. Jochen Steinmann ${ }^{6} \cdot$ Hannelore Willkommen ${ }^{7}$

${ }^{1}$ Institute for med. Virology, University Hospital Frankfurt, Frankfurt/Main, Germany

${ }^{2}$ Robert Koch Institute, Berlin, Germany

${ }^{3}$ Paul-Ehrlich-Institut, Langen, Germany

${ }^{4}$ Laboratory Prof. G. Enders MVZ GbR, Stuttgart, Germany

${ }^{5}$ Laboratory Dr. Merk \& Colleagues, Ochsenhausen, Germany

${ }^{6}$ Dr. Brill \& Partner GmbH Institute for Hygiene and Microbiology, Bremen, Germany

${ }^{7}$ RBS Consulting, Erzhausen, Germany

\title{
Comment on the significance, application and determination of the large volume plating (LVP)
}

\section{Communication of the DVV/GfV Virus Disinfection Expert Committee on the DVV/RKI Guideline in the version of December 1st, 2014}

\author{
In which cases may or should \\ the LVP be applied?
}

\section{Section 6 "Determination of the infectivity of samples in the suspension test"}

This section emphasizes that the LVP should only be applied under certain conditions. This procedure reflects the standard method for infectivity determination as applied in many laboratories. Irrespective of this, it is theoretically possible to apply alternative methods for quantitative determination and calculation of the infectivity.

The LVP is usually carried out in case of high cytotoxicity and low virus titers (in such cases the correct calculation of the virus titer according to Spearman and Kärber is often not applicable because the first dilution used does not produce a high reaction rate, i.e. only a part of the cultures is positive.) The use of the LVP is described in the Guideline in a very strict manner.

In section 6, paragraph 4 it says:
So-called "large-volume-plating" (LVP) is another method that can be used to improve or refine detection of a decrease in infectivity at the end of the contact time with the disinfectant. This method must only be applied if it is impossible to reach a titre reduction of $\geq 4 \log _{10} u$ sing the previously described method (end point dilution method without cytotoxicity reduction measures) due to reasons described in the previous paragraph. LVP improves the detection limit and can only be used if no or very little infectivity can be demonstrated in the infectivity tests (end point dilution method or plaque assay).

Actually, it is not mandatory to test the viral load at first according to the end point dilution method or the plaque test before testing a larger sample volume according to the LVP.

Therefore, it is theoretically possible that the infectivity can be determined by the LVP method exclusively. The Guideline recommendation for testing the viral load at first according to the end point dilution method or the plaque test prior to testing a larger sample volume according 
to the LVP, complies with the standard practice of limiting the end point dilution range of the sample. The determination of viral loads according to the LVP is generally possible, if the infectivity of the sample lies within the end point dilution range. If all cavities of the micro test plates used are positive, no result will be reached.

If the infectivity of the sample is determined exclusively by LVP, a statistical evaluation is required.

\section{Calculation of the infectivity according to the LVP}

For the calculation of the infectivity according to the LVP, the Guideline states:

The amount of plates and thus the test volume applied determines the detection limit.

The test volume, i.e. the sample extracted from the test cycle and usually applied diluted, determines the detection limit. For example, if $3.5 \mathrm{ml}$ are extracted, diluted $1: 20$ and if this volume $(70 \mathrm{ml})$ is tested on 700 cell cultures in 96-well micro titer plates $(100 \mu \mathrm{l} /$ well $)$, then a better detection limit will be reached than if only $1 \mathrm{ml}$ was extracted and tested with $20 \mathrm{ml}$ extracted from the dilution.

Furthermore, the Guideline states (in section 6, paragraph 4 and section 8.3):

After incubation under specific virus relevant titration conditions, the cell cultures are examined microscopically for cytopathic effects. The virus concentration or rather the detection limit respectively is calculated from the number of infected cell cultures or from the total sample volume used showing no infectivity. That calculated value is then used for the calculation of the reduction factor (see item 8 ).

If some viral infectivity is still found using the LVP method, the virus concentration may be calculated according to the following formula. The formula is derived from the Taylor series and approximating exponential functions (Taylor formula). The result, converted into a logarithmic value, is titer $b$ and is used for the calculation of the reduction factor.
One TCID $50 / m l$ is equivalent to 0.69 infectious virus particles.

$$
c=\frac{D}{V_{W}}\left(-\ln \frac{n-n_{p}}{n}\right)
$$

Key

$c=$ concentration of the infectious virus particles

$D=$ dilution

$V w=$ volume per well

$n=$ number of inoculated wells

$n p=$ number of virus-positive wells

The calculation according to Lycke et al. [2] provides equivalent results (concerning the Taylor formula), so it may equally be applied for calculation. Therefore, expert opinions previously based on the Lycke formula, remain valid. Nevertheless, a uniform calculation basis is targeted; i.e. the calculation should preferablybe performed according to the Taylor formula as mentioned in the guideline.

\section{Calculation in case no residual virus can be identified: Is it possible to apply an alternative formula in case of a negative test result for the calculation of the detection limit?}

In case no infected cell cultures are identified using the LVP, the guideline specifies the use of the formula according to Poisson. The Guideline states in section 8.3:

If no virus is detected using the LVP method, the Taylor formula is invalid and therefore the Poisson formula must be applied.

It includes the statistical distribution of few virus particles in a large volume. It is the calculation of the virus concentration necessary to receive a positive result for a given sample volume with a probability of $95 \%$. The number of virus particles is calculated according to the following formula, which, in consideration of the dilution factor and converted into a logarithmic value, represents titer $b$ and is used for the calculation of the reduction factor:

$$
\begin{aligned}
& p=e^{-C V} \text { and derived from } c \\
& c=\ln p /-V
\end{aligned}
$$

Key

$p=$ is the probability of not detecting a virus; the probability of not finding a virus must not be higher than $5 \%(p=0.05)$ so that the number of virus particles is calculated which can be detected with a probability of $95 \%$

$c=$ concentration of infectious virus particles

$V=$ test volume

As an alternative, the following formula could be used taking into account the total volume other than that used in the test:

$$
\text { Titer } / \mathrm{ml}=\ln p / \mathrm{V} \times \ln (1-\mathrm{v} / \mathrm{V})
$$

Key

$p=0.05$

$\mathrm{v}=$ tested volume

$\mathrm{V}=$ sample volume

This formula is derived from the formula mentioned in the Guideline. In the same manner as for the simplified formula mentioned in the Guideline, this calculation results in the concentration of virus particles extracted from the test cycle and possibly diluted for the test, which had to be present from a statistical point of view to be detected with a probability of $95 \%$.

Therefore, the correct definition of 'sample volume' is important. Used correctly, this is the sample volume obtained from the test cycle and not the volume of the diluted sample applied in the test.

Applied correctly, both formulas may be used for the calculation. For reasons of comparability or evaluation of the results, the formula specified in the Guideline should be applied. 


\section{Corresponding address}

\section{Maren Eggers}

Laboratory Prof. G. Enders MVZ GbR

Rosenbergstraße 85, 70193 Stuttgart, Germany

eggers@labor-enders.de

Conflict of interest. H.F. Rabenau, I. Schwe-

bke, J. Blümel, M. Eggers, I. Rapp, J. Steinmann and

H. Willkommen declare that they have no competing

interests.

\section{References}

1. Rabenau HF, Schwebke I, Blümel J et al (2015) The Guideline of the German Association for the Control of Virus Diseases (DVV) and the Robert Koch Institute (RKI) for testing chemical disinfectants regarding their efficacy in fighting viruses within the field of human medicine. Bundesgesundhbl 58:493-504. https://doi.org/ 10.1007/s00103-015-2131-8

2. LyckeE, Melen B, Wrange G (1957) Studies of the inactivation of poliomyelitis virus by formaldehyde. Arch Gesamte Virusforsch 7(4):378-383 